\title{
Active Balancing Reflexes for Small Humanoid Robots
}

\author{
Sara McGrath \\ Department of Computer Science \\ University of Manitoba \\ ummegratecs.umanitoba.ca
}

\author{
Jacky Baltes \\ Department of Computer Science \\ University of Manitoba \\ jackyecs.umanitoba.ca
}

\author{
John Anderson \\ Department of Computer Science \\ University of Manitoba \\ andersjecs.umanitoba.ca
}

\begin{abstract}
To be practical, humanoid robots must be able to manoeuvre over a variety of flat and uneven terrains, at different speeds and with varying gaits and motions. This paper describes three balancing-reflex algorithms (threshold control, PID control, and hybrid control) that were implemented on a real 8 DOF robot equipped with only an accelerometer sensor to study the capabilities and limitations of various balancing algorithms when combined with a single sensor. In our extensive tests, the basic threshold algorithm proves the most effective overall. All algorithms are able to balance for simple tasks, but as the balancing required becomes more complex (ie, controlling multiple joints over uneven terrain), the need for more sophisticated algorithms becomes apparent.
\end{abstract}

\section{INTRODUCTION}

For humanoid robots to move from fantasy to reality, they must be able to move over a variety of uneven terrain with different speeds and gaits. Useful robots must be able to move about freely, but at present do little in the way of active balancing. The technical challenge at RoboCup 2005 involved a section of uneven terrain, requiring robots to navigate a field with at most $3 \mathrm{~mm}$ differences between each hexagon block in the field. Only Team Osaka's Vision robot crossed the field, without reference to any balancing sensors, simply using a very well tuned walk and foot extensions to lessen the slope [5]. Integrating dynamic balancing into robots will allow them to not only deal with changing surfaces, but also allow them to compensate for sudden changes in their equilibrium. Further, balancing will also allow robots to move to new gaits and tasks, such as crawling or load-bearing with greater ease, and more robustness.

Humans themselves use multiple sensors to balance: vision, position/force feedback (muscle feedback) and tilt/acceleration sensors (inner ear organs). Based on an analysis of the dynamics of a robot, force feedback and motion based sensors have been used by many researchers in combination. However, in contrast most of human balancing occurs subconsciously using balancing reflexes that can be demonstrated by for example tapping a human on the shin. These reflexes are present in any human motion, allowing people to preform basic balancing whatever their actions may be. Little research has been done on simple balancing reflexes that employ a simple algorithm and a single sensor. However, to evaluate the capabilities and limitations of various algorithm and sensor combinations, this type of experimentation is extremely important. Multiple sensors potentially mask or ignore important sensor data.

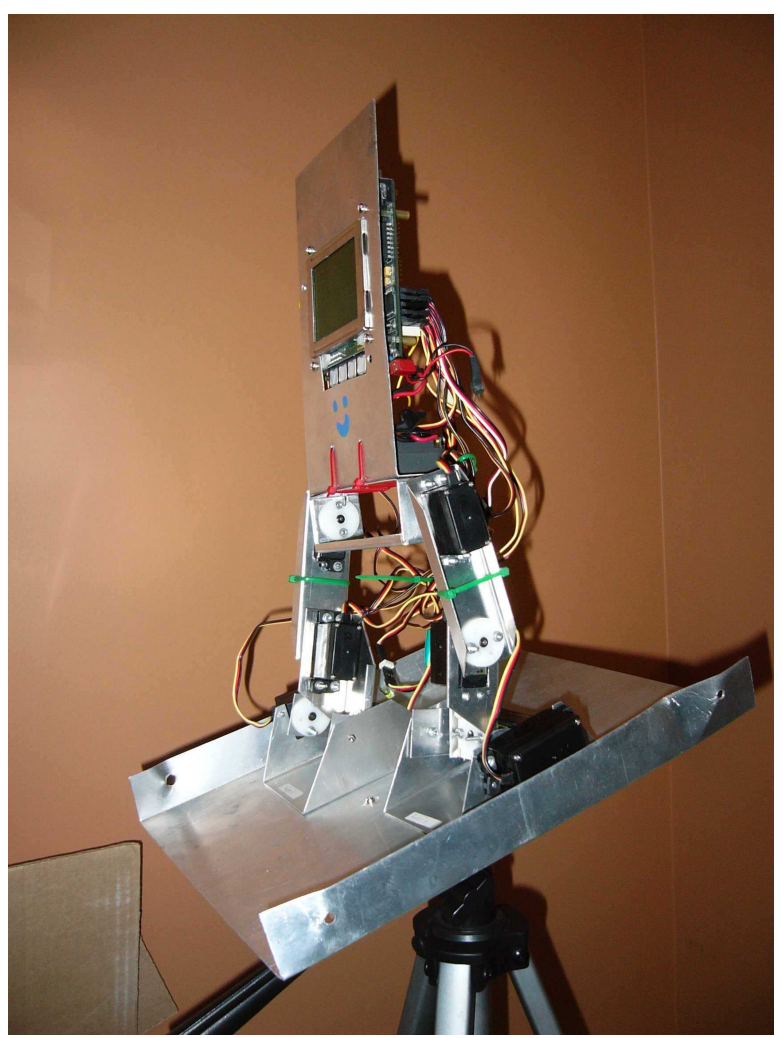

Fig. 1. Lillian, an 8 DOF robot with mounted accelerometer

To develop balancing reflexes, we therefore decided to look at each sensor individually and determine the extent of its usefulness. Thus, an accelerometer was mounted on Lillian, a humanoid robot, as the sole balancing sensor. Lillian, a robot from the University of Manitoba's Autonomous Agents Lab, has 8 DOF (actuated with servo motors), an accelerometer and an Eyebot controller board. The main design principles in building the robot were simplicity and frugality, as this forces the resulting algorithms to be more robust, versatile solutions.

\section{RELATED WORK}

Several special purpose algorithms to adjust a robot's motions are described in the literature. They can broadly be classified into two types: (a) Center Of Mass (COM) based algorithms keep the robot's COM in the supporting polygon of the robot's feet, (b) Zero Moment Point (ZMP) 
algorithms calculate the point in the horizontal plane at which all the moments are zero, and keep the ZMP in the supporting polygon. On the other hand, the versatile PID (Proportional Integral Derivative) controller is a basic control strategy that adjusts the error of a feedback output to a desired reading (baseline), making corrections based on a percentage of the error, and the integral and derivative components of the error. Similarly threshold control, with minimal corrections applied to readings outside a given threshold, was developed in previous research by [4].

Control methods can also be divided into simple balancing reflexes, with tight control loops between sensors and simple algorithms, and more specialized algorithms such as AutoBalancer [2]. Exploration into new sensors tends to begin with simpler control methods and then more complicated algorithms. While these intensive algorithms can use more complicated methods to be fine-tuned (ie, reinforcement learning, genetic algorithms), they apply to the specific robot, and are more computationally intensive to create, often requiring preprocessing. Reflexes use less complicated methods, and are simpler, more general functions that could ideally transfer more effectively than do the highly-tuned algorithms.

Beijing University [1] investigated using sensory reflexes on their robot BHR-01, incorporating a ZMP reflex, a landing phase reflex, and a posture reflex into the dynamic walking pattern. These reflexes were triggered by sensory information, and when active, would compensate for any imbalances in the walk by adjusting the ankles, hips or knees. The corrections were used to adjust the offline pre-calculated walk pattern. These realtime reflexes, added to the walking pattern, proved effective in walking over uneven terrain.

Team KMUTT [3] used a simpler velocity based control to dynamically balance their robot. This balancing mechanism is part of a specific walk, chosen by the robot if its sensors indicate conditions are appropriate, not a standard part of the robot's behaviour. The robot has two PD-controlled walks: a slower static walk that uses the force sensors on the robot, and a faster dynamic walk that balances using accelerometers and gyroscopes. In the static walk, the PD controller manipulates the height at the robot's hip based on the force sensors in the foot. The dynamic walk controls the velocity at the hip with its PD controller. Team KMUTT competed at RoboCup 2006 using this code.

The University of Manitoba's Tao-Pie-Pie is the only robot to use only gyroscope readings for correcting balance. [4] The readings are processed and run through a Threshold controller, compensating for perturbations in the gait. The Threshold controller simply applies a minimal correction when sensor readings break a predefined boundary. Balance is explicitly added on as corrections made to the precalculated walk gait. These corrections were used in competition to compensate for the poor surface, and were found to be better than the previous gait. Further, the Threshold method is extremely simple to implement and tune.

\section{MethodOLOGY}

Three of the many potential algorithms were used here to convert sensory readings into motion corrections and thus implement a balancing reflex: a standard PID controller, a threshold based controller, and a hybrid version of the two. For an accelerometer based PID control, a baseline is created by either taking a sample from previously programmed good motions (eg, a walk gait), or setting the baseline to be unmoving (stand). The closer the baseline conforms to the actual readings, the better the corrections. Threshold balancing first determines a threshold area, where no corrections will be made. This threshold is currently centered on the PID baseline, but with a broader range to avoids corrections from causing oscillation when the robot is standing still. Corrections are only applied once they pass a certain error value. This allows for a simpler means of adjusting settings, and eventual comparison or combination with PID settings. Both methods listed above have their own faults: PID, a quick reaction but a tendency to overcorrect, and Thresholds less of a tendency to overcorrect, but relatively unable to react quickly to larger errors. Thus a hybrid method was theorized and implemented to combine the best of both methods. It uses Thresholds for smaller corrections, but PIDbased corrections for larger errors.

In a model-based approach, the researcher creates a mathematical model of the robot to work on. Then a control algorithm is implemented and tested on the model (often in simulation). Finally, the controller is moved to the physical robot. Often, some readjustments are required at this point to transfer the controller. One of the disadvantages of this method is the fact that it is often difficult to develop a mathematical model for an existing robot that is accurate enough to help develop balancing algorithms.

In contrast our approach is implementation-based and does not depend on a model of the robot. The robot itself is used as a test platform. This removes the necessity of adjusting the control strategy for the robot or unforeseen physical factors, as the testbed is perfectly accurate. The drawback is in maintaining the robot and the length of time required for each test. Our methodology is to modify a pre-existing gait to improve it. Thus, our control algorithms are applied to the current gait, allowing the results of each strategy to be directly compared with each other and the uncorrected walk on the real robot.

Therefore, Lillian's servos are controlled using key control points, linearly interpolation to determine the current placement of the servo in question. These control points are created from an initial position, with changes made by position adjustments. This allowed gaits to be easily adjusted for new robots, modified hardware, or corrections based on sensor input by changing the initial stance or modifying the adjustments. Sensor data is obtained by filtering the accelerometer readings using a running average, and calibrating a zero point, allowing walks to be compared to each other. These readings are used by a correction method to create corrections for the gait that directly modify the servo 
movements. For Lillian, corrections are made to each set of joints, for each axis, individually.

The first step in correcting the gait is to calibrate a gait by creating a baseline, the desired reading or reading range from the sensor input. Generally, a baseline is created from a known good gait which is stable and repeatable. Sensor readings over multiple steps create a band that can be copied for a baseline. Future walks can then be manipulated to follow the baseline, regardless of the terrain. As a general rule, the more accurate the baseline is to a good walk created on the robot in its current physical state, the better the corrections. Experience has shown that tuning is less tied to a particular gait than calibration. Once the robot has been properly calibrated for a gait, physical repairs to the robot such as servo replacement will alter the tuning very little.

The Sum of Absolute Error (SAE) is a quantitative measurement used here to determine the relative goodness of varying walks. As all the correction methods have a baseline (or a set of thresholds), the deviations can be measured to directly compare trials. Summing the absolute errors measures the total deviations from the baseline. The greater the total deviation, the less the walk conforms to the baseline, and the less the corrections are helping.

As with most balancing approaches, Lillian was tuned for one plane before complicating matters with multiple planes, inclines, or uneven terrain. Further, as differing gait disturbances produce oscillation at differing points in the tuning, small increments of complexity are necessary to allow for the robot to be properly tuned. As many researchers use only one plane, or do not specify a method by which to tune for increasingly complicated balancing, one is given here.

Tuning begins with standing still, before moving to tilting, walking, and then multiple joints and planes. First, the corrective methods are tuned not to oscillate during stands. This gives a minimal value to use as a base for the PID settings and threshold bounds. The next step is for the robot to stand still on a surface that tilts in either the frontal or sagittal plane, thus forcing corrections for deviations from its desired pattern. In order to minimize possible complicating factors, the robot will correct in one axis at a time, attempting to maintain a sensor reading of zero. Once Lillian can remain stable while tilting, a more complicated sensor pattern can be used, and the tuning adjusted. Following a walk baseline on a flat surface instead of a steady line adds another level of complexity to the balancing, exposing previously hidden oscillation, as the balancing must compensate at many levels and speeds. Balancing in two axes is much more difficult than any of the previous tasks, as any oscillation (or even a too quick correction) in one axis can produce a rebound and perhaps oscillation in the other axis. Thus, two axes are not tested until balancing is working effectively in one axis. Again, two axes balancing starts with a simple tilting platform before moving to a more complicated pattern walking on a even surface, then uneven terrain. Tuning is limited here to walking on a flat surface. Further tests are used to evaluate the algorithms.
While this methodology only uses a walk gait for its most complicated sensor pattern, any gait will have a repetitive pattern that can be used to calibrate a baseline. These gaits include crawling and running, to begin with. Once Lillian has been tuned for multiple planes of balancing, it is possible to replace the walk baseline with one created from a crawling gait, for instance. Corrections would then be made based on the new gait, but use the same tuning as the previous gaits. This allows the balancing reflexes implemented here to transfer quickly and easily to new gait patterns.

Tuning configuration began with the PID and threshold methods on the tilting platform. The test results were used to refine the PID and Threshold walking tests. The best results were used to test the hybrid method. A side-by-side comparison of all the best results was used to choose settings for the final tests with the perturbed walks, and the stepping field tests.

Testing on the tilting platform began with the PID and Threshold correction methods applied to individual joints (XAS, XK, and Y), and then the best results were used to create further walking tests. The platform was tilted from $-30^{\circ}$ to $+30^{\circ}$, from a starting position of $0^{\circ}$, with an angular velocity of $240^{\circ}$ per minute. The tests were coarse grained, running a trial with correction values of 150, 450, 750 and 1050, with delays of 1, 4, and 7 timesteps required between corrections. The time delay in between each correction turned out to be much more important than previously realized. Allowing corrections at any time caused corrections to be made to the robot before the effects of previous corrections reached the sensors, resulting in severe oscillation. Both the $\mathrm{X}$ and $\mathrm{Y}$ axis had to be tested for how often corrections could be made. Results from the tilt testing were used to further explore interesting values. Testing began with the threshold method, and results from those tests further pruned the tests run on the PID method, as PID is more likely to overreact, producing oscillation or poor results.

After tuning the $\mathrm{P}$ settings, the best setting from each joint was taken and tested with a range of differing D settings, by setting a baseline that stayed at +1000 for the first half of the gait, and then moved to -1000 for the second half. The only movement was provided by the corrections adjusting the accelerometer readings. It was thus possible to look for the overshoot caused (or avoided) by varying the D parameter, running each trial once for an initial exploration.

Results from the single joint tests were used to tune for correcting multiple joints simultaneously. Results that improved on the baseline where possible, and the best results available otherwise, were selected for further tuning and divided into two (best and good). Each of the best joint settings was paired with each of the best and good settings for another joint, to allow reasonably thorough testing without factorial explosion. Each of these tests were run three times, to reduce the noise in the data. Further, $\mathrm{X}$ joints were first tested and combined with each other (XAS and XK) before adding in a second plane with the $\mathrm{Y}$ ankle joint. This follows the ideology of the prior tests in adding in as little complexity as possible to each test for a clear picture of the effects of 
each new factor.

For D, three trials were run with the best setting for multiple joints tested against a range of the best $D$ settings previously determined. Both $\mathrm{P}$ settings were enabled, and then one D setting at a time was enabled for each range. Finally, both D settings were turned on, and the better of both ranges used simultaneously to compare the benefits of D on multiple joints.

While settings appear non-linear, the test results range from oscillation to undercorrection. In general, control settings affected the walk more than the delay, but a delay between corrections was necessary to prevent oscillation. More settings improved on the tilting than the walking, while no improvement was noticed with corrections applied to multiple joints. Overall, PID produced only a few possible improvements, while Thresholds provided many more choices.

\section{EVALUATION}

A basic walking gait was first used to evaluate the best of the tuning results side by side. Any result that improved on the gait (in the plane it was correcting) was used for the final evaluation. Tests include randomly perturbing the walk to varying degrees, and running Lillian over a stepping field, with and without balancing reflexes, for approximately twenty seconds. Gaits were perturbed by randomly varying the control points of one good gait over a spread of 5 or 10 set points ([-2 .. 2], or [-5 .. 5]), at multiple points throughout the gait. The disturbances were applied to both joints, as the balancing control assumes that the movements of the joints are coupled. A stepping field was constructed of layered pieces of cardboard, always providing a height difference of $3 \mathrm{~mm}$ between neighbouring pieces, but possibly more than one piece over the length or width of Lillian's foot.

The sheer number of tests carried out to tune the controllers on the robot make it difficult to determine what settings best improves the walk. Therefore, the best settings in each method, PID and Threshold, were directly compared against each other, though the SAE is normally calculated differently for both. As the thresholds are currently set equidistantly from the PID baseline, the SAE was calculated using the PID baseline on all non-tuning trials.

The PID controller settings chosen were the best for each joint, the best combination of settings for the $\mathrm{X}$ joints, the best for all the joints, and the best $\mathrm{P}$ and PD controllers for Y. No D settings were chosen for any of the $\mathrm{X}$ joints as they had not improved the controllers. found to improve on the basic walk, no further combinations of settings involving them were chosen.

The Threshold settings were similarly chosen as the best setting for each joint, and the best combinations of $\mathrm{X}$ and $\mathrm{XY}$ joints. Two XY settings were chosen, as one improved on the basic walk for $Y$, and the other was the best set of corrections for $\mathrm{X}$. Two more settings were chosen for testing: $\mathrm{XAS}+\mathrm{Y}$ and $\mathrm{XK}+\mathrm{Y}$, as one of the $\mathrm{XY}$ settings was the same as the three best individual joint settings. Further, each of the single joint settings (unlike the PID controller) actually
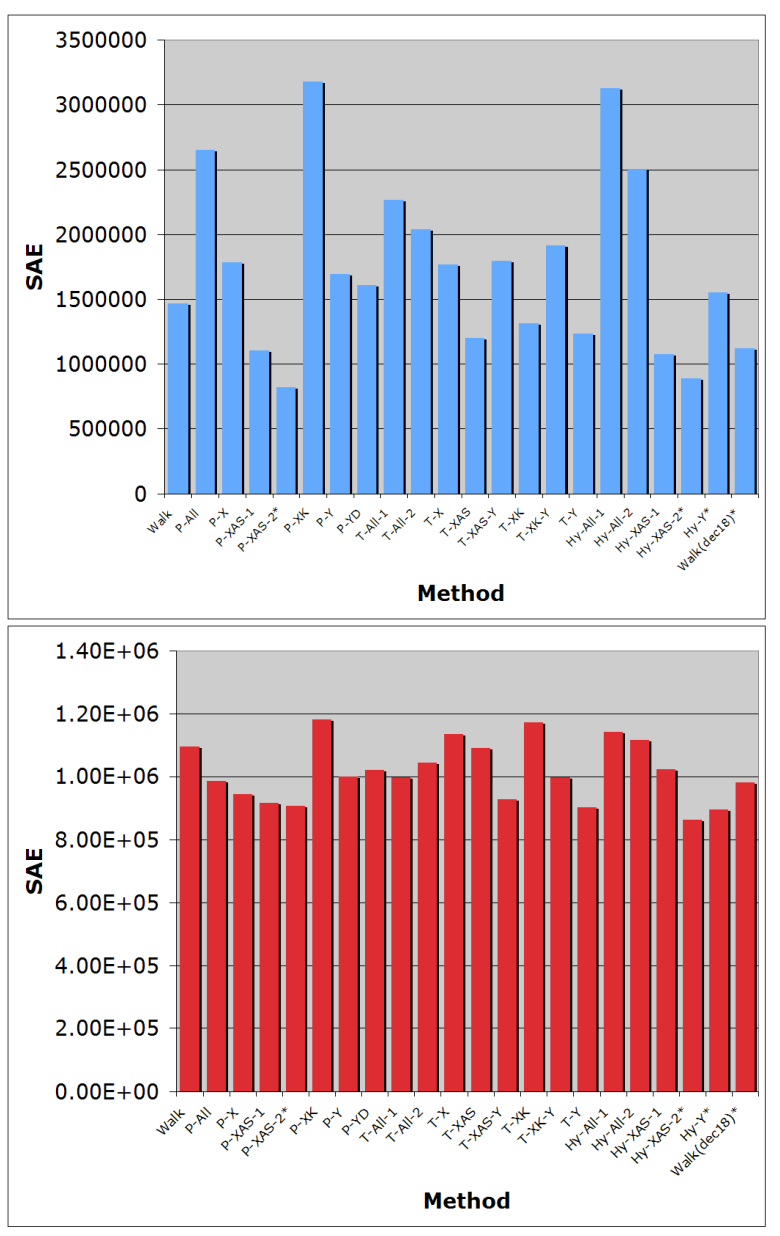

Fig. 2. Random walking test results, by method and settings.

improved on the basic walk, suggesting that a single $X$ joint setting with a Y setting could prove effective.

As the hybrid controller depends on combining the Threshold and PID controllers, it was only tested after the best settings for the simpler controllers were determined. The hybrid controller is tested on settings that improve the walk: on the top two XAS settings for both PID and Threshold; the combination of the Y controllers; and for comparison's sake, the two best Threshold XY controllers with the best PID XY controller.

Figure 2 shows the results of the direct comparison. Less than half actually improve upon the uncorrected walk, in the plane(s) that they are correcting for. This criteria is used to select settings for the final evaluations, giving P-XAS-1, P-XAS-2, Hy-XAS-1, Hy-XAS-2, T-XAS, T-XK, T-Y, P-Y, and $\mathrm{Hy}-\mathrm{Y}$.

\section{A. Random Walks}

As can be seen in the graphs in Figure 3, any correction method makes an incredible difference in the perturbation of the walk. This is best noticed in the Random Walk 5 graphs (Figure 3). The SAE of the methods correcting in the $\mathrm{X}$ plane is a third to a quarter of the uncorrected methods. Solely Y corrections are not as impressive, leaving the walk 


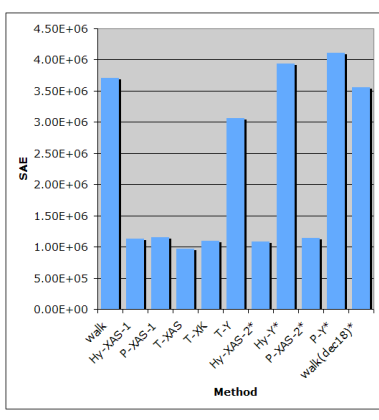

(a) Random Walk 5: X

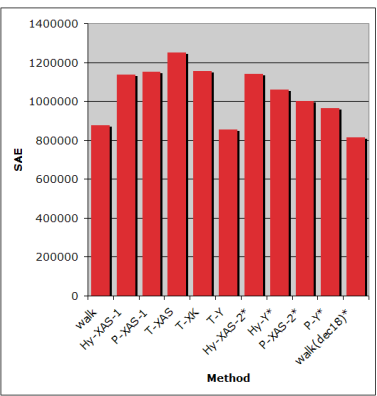

(b) Random Walk 5: Y
Fig. 3. Random walking test results with a perturbation spread of 5, by method and settings.

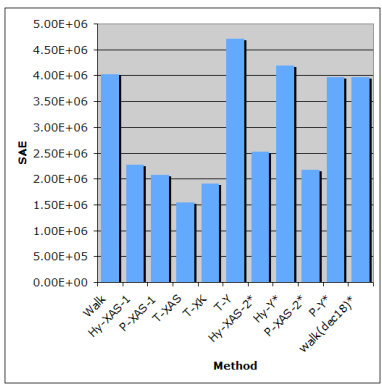

(a) Random Walk 10: $\mathrm{X}$

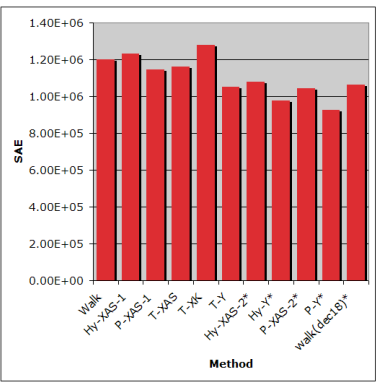

(b) Random Walk 10: Y
Fig. 4. Random walking test results with a perturbation spread of 10 , by method and settings.

unimproved. The differences between correction methods in this test are much less than the difference between the corrected and uncorrected tests. Thresholds have the best results here, then hybrid, then PID, while all improve the walk. For Thresholds, the best joint control is on the XAS joint; the XK joint is not as effective. Threshold control on the $\mathrm{Y}$ joint is not extremely effective; it improves the walk slightly in both planes, but not as much as the $\mathrm{X}$ control. This is partly due to most of the imbalances created by the perturbations arising in the $\mathrm{X}$ plane; the $\mathrm{Y}$ plane, having only one joint to control, is naturally more resistant to disturbances in the walk pattern. Hybrid correction in this case appears to bridge the gap between Threshold and PID: it's not as effective as Threshold, but more effective than PID.

Increasing the perturbations to up to a spread of 10 caused the differences between the methods to become yet more pronounced, as shown in Figure 4. Again, the Threshold results are the best in the $\mathrm{X}$ plane, but with a greater difference between them and the other methods. Any correction method still shows a marked improvement over the uncorrected walk, but the errors overall are larger than they were for the previous test, indicating that the correction methods are not as well able to cope with the error caused by the larger perturbations. Unlike in the $\mathrm{X}$ plane, the PID method is the best, followed by the hybrid method, and finally the Threshold method.

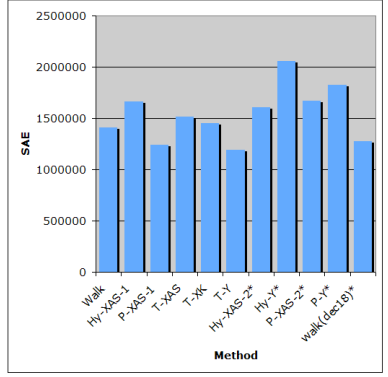

(a) Stepping Field: $\mathrm{X}$

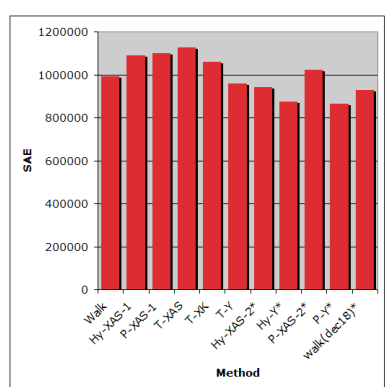

(b) Stepping Field: Y
Fig. 5. Stepping field test results, by method and settings.

\section{B. Stepping Field}

Results from the stepping field (see Figure 5) show that the balancing degrades here. For the X plane, only the PID corrections improve on the uncorrected walk. While the hybrid method actually worsens the SAE readings, Threshold corrections merely leave the walk mostly unchanged. Unlike previous tests, Threshold corrections on the XK joints are actually slightly better than those of the XAS joint. Compared to the differences between the corrected and uncorrected walks in the perturbation test, however, the differences between the results are relatively minor, indicating the $\mathrm{X}$ corrections are not making a large difference to the walk. As expected, the lack of corrections to the $\mathrm{Y}$ plane by these methods is clearly shown, as the $\mathrm{Y}$ readings for all the $\mathrm{X}$ correction methods are worse than the uncorrected $\mathrm{Y}$ walk. The threshold corrections for $\mathrm{Y}$ improve on the uncorrected walk for both $\mathrm{X}$ and $\mathrm{Y}$, not just $\mathrm{Y}$. Therefore, unlike the perturbation test, where the main corrections to be made were to the $\mathrm{X}$ plane, here the most effective corrections are to the $\mathrm{Y}$ plane, and correcting in $\mathrm{X}$ without correcting $\mathrm{Y}$ will not be very effective.

\section{Analysis}

Directly comparing all results with each other led to some interesting observations. The more joints a controller attempted to control, the worse the balancing became. Similarly, more complicated controllers, such as PD instead of P, did not improve the balancing. The simplest ideas, such as the threshold controller, were just as effective in controlling the walk, and maintaining the desired accelerometer readings. The simpler threshold controller also responded better to adding more joints, not overreacting as much as the PID controller.

This conclusion of complexity not being handled well was further born out by the random walks. Different joints were also more effective in controlling the balancing. The XAS joint is much more effective at controlling the robot's balance than any of the other tested joints. Further, as the perturbations increased, Threshold showed as more effective than PID. The hybrid controller is still better than no controller, but not as good as either of the simpler controllers.

The stepping field upsets the previous trends with PID actually outperforming Thresholds. This suggests the threshold 
controller is unable to react quickly and strongly enough for the corrections required. The random walks had a change of perhaps 5 or 10 servo settings over 1 second; the threshold controller allows approximately 10-12 servo setting changes per second. Thus the threshold controller could compensate for the changes in the walk, while the PID controller tended to overreact. Once on the stepping field however, the changes would occur instantly, and require compensation of 5-10 servo settings (or perhaps more). The PID controller could react instantly, while the threshold control would move more slowly to correct. This slower reaction time would allow the robot enough time to accumulate inertia in the wrong direction, making it even more difficult for the robot to compensate. A further explanation of the poorer results on the stepping field is due to the initial assumption that all joint actions are coupled. This is generally true of the testing, as with the randomized perturbations and the tilting, the robot's feet remain relatively aligned with each other. The stepping field, however, due to the unevenness of the terrain, allows for the feet to become misaligned, and this is not easily corrected by the balancing reflex, as currently implemented.

\section{CONCLUSiOnS AND Future WORK}

This research showed that simple reflexes can be used to balance a robot in simple situations, but that they become unable to handle the complexities of more normal situations (ie, walking while controlling multiple joints). Both Threshold and PID algorithms showed impressive results on the tilting tests, with a broad range of settings providing beneficial corrections to the robot. Moving those corrections to a walk demonstrated the shortcomings of the reflex algorithms, as the speed and complexity of balancing a walking robot started to overcome the balancing capabilities of the controllers. This was most noticeable with PID, as Thresholds still had several useful settings. Adding multiple joints to be controlled, or moving the robot to an uneven surface further demonstrated the inability of the balancing reflexes to compensate for the amount of variability in the walk. While the reflexes were not able to fully compensate for any surface, they did improve the walk noticeably against smaller, more regular changes, as shown by the random perturbation tests.

No one algorithm was consistently best; rather, the most effective algorithm depended on the circumstances the robot was used in. Indeed, results were close enough that the strongest conclusion is any controller is better than none. Overall, Thresholds appeared best for slower, steadier changes, while PID responded better to occasional larger changes. The Hybrid method was never the best method, but was almost always in between the two other algorithms in terms of goodness; never the best, but regularly the runnerup. In every test, however, at least one of the correction methods matched or outperformed the uncorrected walk, showing that a tuned correction method is better than no correction.

Overall, due to its ease of tuning and general performance, the threshold method is the easiest and most useful choice for future balancing. While there are differences between the methods, for a single joint they all do improve upon the uncorrected walk, and thus any method is better than none.

This research has focused on a walk gait; this should be expanded to include more complex motions such as crawling or load-bearing in future. The simple reflexes used here can apply directly to a different gait (such as crawling) by simply calibrating the crawl to give Lillian a new baseline, and applying the same corrections used for the walk on a crawl. Load-bearing is even simpler, as it relates to a previous motion. Changes should not have to be made to the robot, but simply weights added to the robot, and the balancing reflexes should immediately begin to compensate for the extra weight.

Future work should address the initial assumption was that all joint movement would be coupled, investigating the possibilities and difficulties involved with allowing each foot or leg to be corrected separately. Differing threshold bounds were used in prior work [4], but have not been investigated here. They may improve the corrections made by the threshold algorithm, as it allows for corrections to be more or less sensitive without extra tuning. Finally, physical modifications to the robot could make it more difficult for the robot to balance, such as by adding weight at the head for extra sway.

This research provides an initial foundation for work looking into balancing reflexes with accelerometers, as it shows that it is possible to balance with only an accelerometer, and a simple control method. However, it also shows that these methods only work for reasonably simple balancing. More complex adjustments are not implemented well with these methods. Terrain such as the stepping field will require in addition more complicated (or at least more effective) means of balancing than either a PID or a threshold controller.

\section{REFERENCES}

[1] Qiang Huang, We ming Zhang, and Kejie Li. Sensory reflex for biped humanoid walking. In Proceedings of the 2004 International Conference on Intelligent Mechatronics and Automation, 2004.

[2] S. Kagami, F. Kanehiro, Y. Tamiya, M. Inaga, and H. Inoue. Autobalancer: An online dynamic balance compensation scheme for humanoid robots. In Proceedings of the Fourth International Workshop on Algorithmic Foundations on Robotics, 2000.

[3] Pasan Kulvanit, Bantoon Srisuwan, Khunit Siramee, Apinya Boonprakob, and Djitt Laowattana. Team kmutt: Team description paper. In Proceedings of the RoboCup 2006, 2006. Accessible at www.humanoidsoccer.org/qualification/KMUTT_TDP.pdf; accessed December 12, 2006.

[4] S. McGrath, J. Baltes, and J. Anderson. Active balancing using gyroscopes for a small humanoid robot. In S. C. Mukhopadhyay and G. Sen Gupta, editors, Second International Conference on Autonomous Robots and Agents (ICARA), pages 470-475. Massey University, December 2004.

[5] Nobuo Yamato, Yohei Akazawa, Hiroshi Ishiguro, Tomotaka Takahashi, Takeshi Maeda, Takuro Imagawa, Hitoshi Takayama, Noriaki Mitsunaga, and Takahiro Miyashita. Teamosaka. In RoboCup 2005 Proceedings CD, 2005. 